\title{
Proper motions and membership probabilities of stars in the region of globular cluster NGC $6809^{\star, \star \star}$
}

\author{
D. P. Sariya ${ }^{1}$, R. K. S. Yadav ${ }^{1}$, and A. Bellini ${ }^{2}$
}

\author{
1 Aryabhatta Research Institute of Observational Sciences, Manora Peak, 263129 Nainital, India \\ e-mail: [devesh;rkant]@aries.res.in \\ 2 Dipartimento di Astronomia, Universita di Padova, Vicolo dell' Osservatorio 2, 35122 Padova, Italy \\ e-mail: andrea.bellini@unipd.it
}

Received 29 March 2012 / Accepted 9 May 2012

\begin{abstract}
Context. NGC 6809 is a luminous metal-poor halo globular cluster that is relatively easy to study due to its proximity and low concentration. Because of its high Galactic latitude $\left(b=-23^{\circ}\right)$, interstellar reddening and contamination is not very high.

Aims. We aim to determine the relative proper motion and membership probability of the stars in the wide area of globular cluster NGC 6809. To target cluster members reliably during spectroscopic surveys and both spatial and radial distributions in the cluster outskirts without including field stars, a good proper motion and membership probability catalogue of NGC 6809 is required.

Methods. The archival data of two epochs with a time-base line of 7.1 years have been collected with Wide Field Imager (WFI) mounted on the $2.2 \mathrm{~m} \mathrm{MPG/ESO}$ telescope. The CCD images of both epochs have been reduced using the astrometric techniques as described in Anderson et al. (2006). The calibrated UBVI magnitudes have been derived using Stetson's secondary standard stars. Results. We derived the relative proper motion and membership probabilities for $\sim 12600$ stars in the field of globular cluster

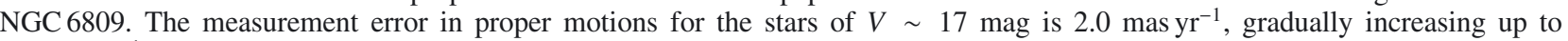
$\sim 3 \mathrm{mas} \mathrm{yr}^{-1}$ at $V=20 \mathrm{mag}$. We also provide the membership probability for the published different types of sources in NGC 6809 . An electronic catalogue with proper motion and membership probability for the stars will be available to the astronomical community.
\end{abstract}

Key words. globular clusters: individual: NGC 6809

\section{Introduction}

Globular clusters have long been used to study the structure and formation of our Galaxy. NGC 6809 (M 55) is a sparse, metalpoor globular cluster whose proximity $(\sim 5 \mathrm{kpc})$ makes it an excellent target for an in-depth study of its stellar population.

NGC 6809 harbours several interesting objects. Bassa et al. (2008) found $16 \mathrm{X}$-ray sources within the half-mass radius (2'89) of NGC 6809, of which eight or nine are expected to be background sources. On the basis of optical counterparts, these authors identified three sources related to the cluster. The brightest X-ray source of this cluster is classified as a dwarf nova. Blue stragglers in NGC 6809 have been studied by Lanzoni et al. (2007) and exhibit a bimodal radial distribution with a central peak, a broad minimum at intermediate radii, and an upturn outwards. A detailed study about the evolved stars, including asymptotic giant branch, horizontal branch, and upper red giant branch (RGB) stars was presented by Vargas et al. (2007).

A proper-motion (PM) study of NGC 6809 was conducted by Dinescu et al. (1999) using photographic plates. The authors determined an absolute proper motion $\left(\mu_{\alpha} \cos \delta=-1.57 \pm\right.$ $\left.0.62 \mathrm{mas} \mathrm{yr}^{-1}, \mu_{\delta}=-10.14 \pm 0.64 \mathrm{mas} \mathrm{yr}^{-1}\right)$ using a sample

* Based on observations with the MPG/ESO $2.2 \mathrm{~m}$ and ESO/VLT telescopes, located at La Silla and Paranal Observatory, Chile, under DDT programs 077.D-0372(A), 163.O-0741(C) and the archive material.

$\star \star$ Full Table 5 is only available at the CDS via anonymous ftp to cdsarc.u-strasbg.fr (130.79.128.5) or via

http://cdsarc.u-strasbg.fr/viz-bin/qcat?]/A+A/543/A87 of $\sim 600$ cluster stars brighter than $V \sim 16$ photographic magnitude and background galaxies as a reference. Recently, a proper motion study of NGC 6809 was performed by Zloczewski et al. (2011, hereafter Z111). They determined membership probabilities for 16645 stars in the central part of the cluster $(8.83 \times$ 8.83) and found an absolute proper motion $\mu_{\alpha} \cos \delta=-3.31 \pm$ $0.10 \mathrm{mas} \mathrm{yr}^{-1}$ and $\mu_{\delta}=-9.14 \pm 0.15$ mas yr$^{-1}$.

Despite the extensive photometric studies of this cluster, there is a lack of studies that provide proper motions and membership probabilities in the wide field region for NGC 6809. Wide-field images allow us to map completely any open or globular cluster in our Galaxy and its tidal trails and allow us to obtain accurate photometry for an enormous number of stars. In combination in photometric data, membership information is very useful to keep the field star contamination to a minimum. The archival wide-field multi-epoch observations taken with the WFI/2.2 m telescope offer new opportunities to derive precise PMs with only a few years of time span, deeper by several magnitudes than previous photographic surveys (Anderson et al. 2006; Yadav et al. 2008; Bellini et al. 2009).

The main purpose of the present study is to determine accurate relative PMs and membership probabilities for stars brighter than $V \sim 20 \mathrm{mag}$ in the wide area of NGC 6809. Membership probabilities of different sources in NGC 6809 are also discussed. Fundamental parameters of the cluster taken from Harris (1996) are listed in Table 1. The PMs, membership probabilities, and photometric $U, B, V$ and $I$ magnitudes are provided to the astronomical community for follow-up studies. Our membership-probability catalogue is in wide field $\left(26^{\prime} \times 22^{\prime}\right)$ 
Table 1. Fundamental parameters of NGC 6809 taken from Harris (1996).

\begin{tabular}{lc}
\hline \hline Parameters & Values \\
\hline$\alpha(\mathrm{J} 2000)$ & $19^{\mathrm{h}} 39^{\mathrm{m}} 59^{\mathrm{s}} 4$ \\
$\delta(\mathrm{J} 2000)$ & $-30^{\circ} 57^{\prime} 44^{\prime \prime}$ \\
$l$ & 8.80 \\
$b$ & $-23^{\circ} .27$ \\
{$[\mathrm{Fe} / \mathrm{H}]$} & -1.81 \\
$E(B-V)$ & 0.08 \\
$(m-M)$ & 13.87 \\
\hline
\end{tabular}

Table 2. Description of the WFI/2.2 m data sets.

\begin{tabular}{|c|c|c|c|}
\hline Filters & $\begin{array}{l}\text { Exposure time } \\
\text { (in seconds) }\end{array}$ & Seeing & Airmass \\
\hline & 1999 May-July & & \\
\hline$B$ & $2 \times 240$ & $1 . \prime 0$ & 1.0 \\
\hline V/89 (First epoch) & $4 \times 200$ & 1.1 & 1.1 \\
\hline$I / l w p$ & $7 \times 150 ; 1 \times 200$ & 1.0 & 1.1 \\
\hline & 2006 August & & \\
\hline U/50_ESO 877 & $6 \times 300$ & 1.1 & 1.0 \\
\hline$V / 89$ (second epoch) & $6 \times 40$ & 1.0 & 1.0 \\
\hline
\end{tabular}

Notes. The first epoch data were observed in May and July, 1999, while second epoch data were observed in August, 2006.

and contains $U B V I$ magnitudes, while Z111 have provided membership probabilities only in $V$ magnitude and in the central area of $8.83 \times 8.83$.

The structure of the article is as follows. Data taken for the present study, their reduction procedures and comparison of photometric and astrometric data are described in Sect. 2, where we also determine PMs and differential chromatic refraction. In Sect. 3 we present the cluster membership analysis. In Sect. 4 we use our catalogue to confirm the membership of previously found variables, blue stragglers, and X-ray sources. Finally, Sect. 5 describes the catalogue while Sect. 6 represents the conclusions of the present study.

\section{Archival data and reductions}

Proper motions of the stars in the cluster region were computed using archive WFI/2.2 m V filter images ${ }^{1}$ taken in 1999 (first epoch) and in 2006 (second epoch). The 1999 observing run consists of two images in $B$ filter, taken in May, while four images in $V$ and eight images in $I$ were taken in July. Six $U$-band images and six $V$-band images were acquired in August, 2006.

The WFI/2.2 m consists of eight $2048 \times 4096 \mathrm{EEV} \mathrm{CCDs}$ with 0.238 pixel $^{-1}$ resulting in a total field-of-view $34^{\prime} \times 33^{\prime}$. The observational log with epoch information is listed in Table 2. Images used in the analysis were taken under similar seeing $\left(\sim 1^{\prime \prime}\right)$ and airmass conditions. Long and short exposures were acquired to map the brighter and fainter stars of the cluster. The data used in this article were not collected with the aim of proper motion study.

\subsection{Astromeric and photometric reductions}

The reduction procedures described in Anderson et al. (2006, Paper I) were adopted for the WFI/2.2 m CCD images. This includes de-biasing, flat-fielding, and correction for cosmic rays. In Paper I we showed that the WFI/2.2 m PSF changes

\footnotetext{
1 http://archive.eso.org/eso/eso_archive_main.html
}

significantly with position on the detector. Because of this, an array of empirical point spread functions (PSFs) were constructed for each image to obtain the positions and fluxes of the objects. These PSFs are saved in a look-up table on a very fine grid. To select suitable stars for the PSFs, we developed an automatic code (see Paper I). An iterative process is designed to work from the brightest down to the faintest stars and find their precise position and instrumental flux for $U, B, V$ and $I$ exposures.

In Paper I we showed that WFI/2.2 $\mathrm{m}$ has a large geometric distortion, i.e. the pixel scale is changing across the field of view. To derive the correction for the geometric distortion, we parametrized the distortion solution by a look-up table of corrections for each chip that covered $2048 \times 4096$ pixel each, sampling every 256 pixels. This resulted in a $9 \times 17$ element array of corrections for each chip. At any given location on the detector, a bi-linear interpolation between the four closest grid points of the look-up table provided the corrections for the target point. The derived look-up table may have a lower accuracy on the edges of a field because of the way the self-calibration frames were dithered (see Paper I). An additional source of uncertainty is related to a possible instability of distortions for the WFI/2.2 $\mathrm{m}$ reported earlier. This prompted us to use the local-transformation method to derive PMs. Detailed descriptions about the distortion solution are given in Paper I.

In the local transformation approach a small set of local reference stars is selected around each target object. It is advantageous to use pre-selected cluster members to form a local reference frame because of the much lower intrinsic velocity dispersion among the cluster members. Then, six-parameter linear transformations are used to transform the coordinates from one frame into another, taken at different epochs. The residuals of this transformation characterize relative PMs convolved with measurement errors. In essence, this is a classical "plate pair" method but extended to all possible combinations of the firstand second- epoch frames. The relative PM of a target object is an average of all displacement measurements in its local reference frame. The last step is to estimate the measurement errors from intra-epoch observations where PMs have a zero contribution. A complete description of all steps leading to PMs is given in Paper I.

\subsubsection{Photometric calibration}

In order to transform instrumental $U, B, V, I$ magnitudes into the standard Johnson-Cousin system, we used a list of secondary standard stars provided by Stetson ${ }^{2}$ for $B, V$ and $I$ and by Kaluzny et al. (2005) for $U$ filter. In total, 851 common stars were found in Stetson's catalogue and were used to calibrate $B, V$ and $I$ magnitudes. These common stars have a brightness range of $12.9 \leq V \leq 20.6 \mathrm{mag}$ and a colour range of $-0.5 \leq(V-I) \leq 2.0 \mathrm{mag}$, which coveres all stars brighter than $V=20.0$ mag. 1205 common stars brighter than $V=19$ mag were found in the Kaluzny et al. (2005) catalogue. These stars have a colour range of $-0.5 \leq(U-B) \leq 1.3 \mathrm{mag}$.

We derived photometric zero-points and colour terms using the following transformation equations:

$$
\begin{aligned}
U_{\text {std }} & =U_{\text {ins }}+C_{u} *\left(U_{\text {ins }}-B_{\text {ins }}\right)+Z_{u} \\
B_{\text {std }} & =B_{\text {ins }}+C_{\mathrm{b}} *\left(B_{\text {ins }}-V_{\text {ins }}\right)+Z_{\mathrm{b}} \\
V_{\text {std }} & =V_{\text {ins }}+C_{v} *\left(V_{\text {ins }}-I_{\text {ins }}\right)+Z_{v} \\
I_{\text {std }} & =I_{\text {ins }}+C_{i} *\left(V_{\text {ins }}-I_{\text {ins }}\right)+Z_{i},
\end{aligned}
$$

2 http://cadcwww.hia.nrc.ca/standards/ 
D. P. Sariya et al.: Astrometry of NGC 6809

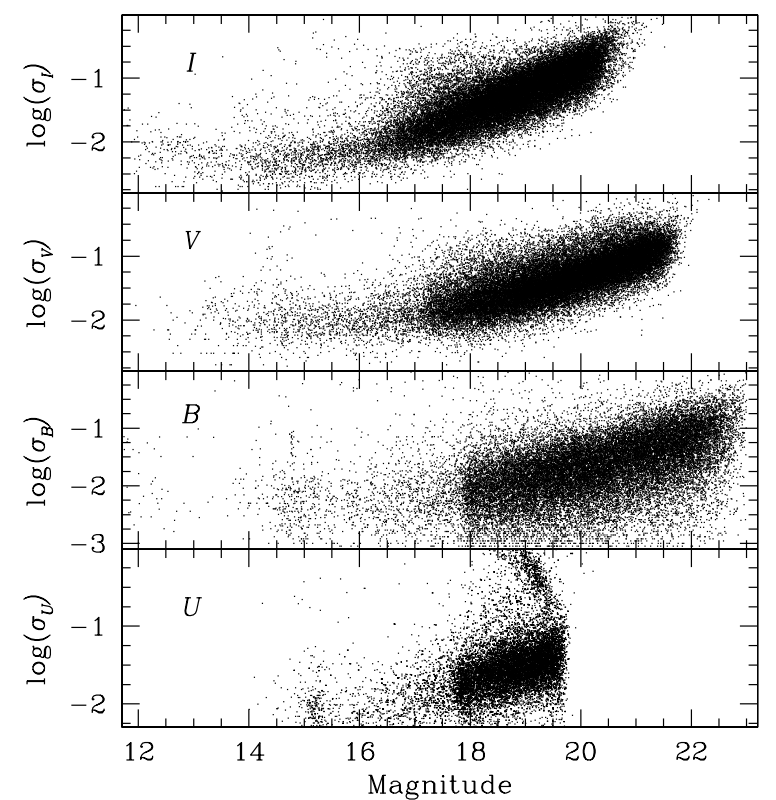

Fig. 1. Plot of the rms logarithm of the residuals around the mean magnitudes in $U, B, V$ and $I$ as a function of their magnitudes.

where the subscript "ins" means instrumental magnitudes and "std" stands for secondary standard magnitudes. $C_{u}, C_{\mathrm{b}}, C_{v}$ and $C_{i}$ are the colour terms while $Z_{u}, Z_{\mathrm{b}}, Z_{v}$ and $Z_{i}$ are the global zero-points. The quadratic colour terms are negligible. The values of colour terms are $0.00,0.70,0.00$, and 0.11 , while the zeropoints are 22.68, 24.95, 24.18, and 23.34 for $U, B, V$ and $I$ filters respectively. Our colour terms and zero-point values are nearly consistent with those posted on the WFI/2.2 $\mathrm{m} \mathrm{webpage}^{3}$.

In Fig. 1 we show the log of rms of the residuals around the mean magnitude for each filter as a function of the respective magnitudes. The photometric standard deviations were computed from multiple observations, all reduced to the common photometric reference frame in the chosen bandpass. On average, photometric rms are better than $\sim 0.03$ mag for stars brighter than 18.0 mag in $U, B$ and $V$ filters. Stars brighter than $I=$ 16 mag have photometric rms lower than $0.01 \mathrm{mag}$, gradually increasing to $0.1 \mathrm{mag}$ for $I=20.0 \mathrm{mag}$.

Figure 2 exhibits the magnitude difference between our calibrated $B, V$ and $I$ magnitudes and the Stetson secondary standards as a function of $V$ magnitude. The difference between our calibrated $U$ magnitudes and the Kaluzny et al. (2005) data is also shown as a function of $V$ magnitude. There are no clear systematic trends seen in the differences with $V$ mag.

\subsubsection{Astrometric calibration}

The next step is to transform $X$ and $Y$ coordinate to right ascension (RA) and declination (Dec). The $X, Y$ raw positions of each star in each frame were corrected for geometric distortion using the look-up table provided in Paper I, brought into common reference frames by means of six-parameter linear transformations and averaged. To transform the averaged $X$ and $Y$ coordinates into RA and Dec of $\mathrm{J} 2000$, we used the online digitized sky ESO catalogue in skycat software as an absolute astrometric reference frame. Thanks to our accurate geometric-distortion solution and a reasonable stability of the intra-chip positions,

\footnotetext{
3 http://www.ls.eso.org/lasilla/sciops/2p2/E2p2M/WFI/ zeropoints/
}

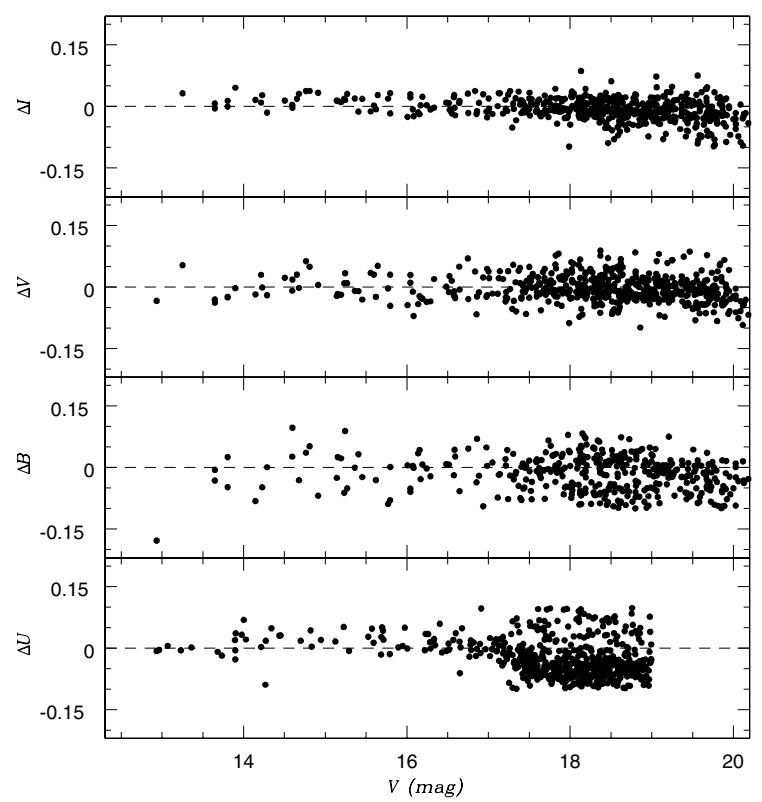

Fig. 2. Star-by-star comparison of our photometry with standard stars. The BVI magnitudes were compared with the Stetson catalogue, while the $U$ filter was compared with data taken from Kaluzny et al. (2005). No clear systematics are present, which proves the reliability of our calibration procedures.

it was possible to apply a single plate model involving linear and quadratic terms and a small but significant cubic term in each coordinate. This solution also absorbs effects caused by differential refraction but not its chromatic component. The standard error of equatorial solution is $\sim 100$ mas in each coordinate.

\subsubsection{Proper motions}

Proper motions were computed using $V$-filter images to minimize colour-dependent terms in our analysis. Moreover, our geometric-distortion solution provides the lowest residuals with $V$ images (Paper I). A total of four images for the first epoch and six images for the second epoch were used.

First, we selected a sample of probable cluster members using the $V$ vs. $(V-I)$ CMD. Selected stars are located on the RGB and $\mathrm{MS}$ in the magnitude range $13.0 \leq V \leq 18.0 \mathrm{mag}$. These stars define a local reference frame to transform the positions of a given first-epoch image into positions of a second-epoch image. By adopting only stars on cluster sequences whose proper

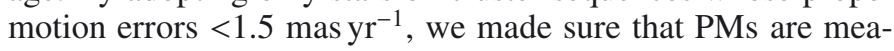
sured relative to the bulk motion of the cluster. To minimize the effect of uncorrected distortion residuals we used the local transformation approach based on the closest 25 reference stars on the same CCD chip. No systematics larger than random errors are visible close to the corners or edges of chips.

We iteratively removed some stars from the preliminary photometric member list that had proper motions clearly inconsistent with cluster membership, even though their colours placed them near the fiducial cluster sequence. According to Pryor \& Meylan (1993) the intrinsic velocity dispersion of stars in the cluster NGC 6809 is $4.9 \mathrm{~km} \mathrm{~s}^{-1}$. With a distance of $5.3 \mathrm{kpc}$ (Harris 1996) the internal proper motion dispersion becomes 0.2 mas $^{-1}$. The distribution log of proper motions errors with $V$ mag is presented in Fig. 3 for both coordinates. The precision of the proper motion measurement (rms of individual measurement) is better than 2 mas $^{-1} r^{-1}$ upto $17.5 \mathrm{mag}$ in $V$.

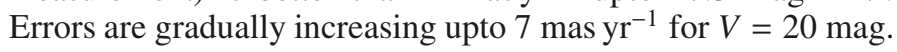




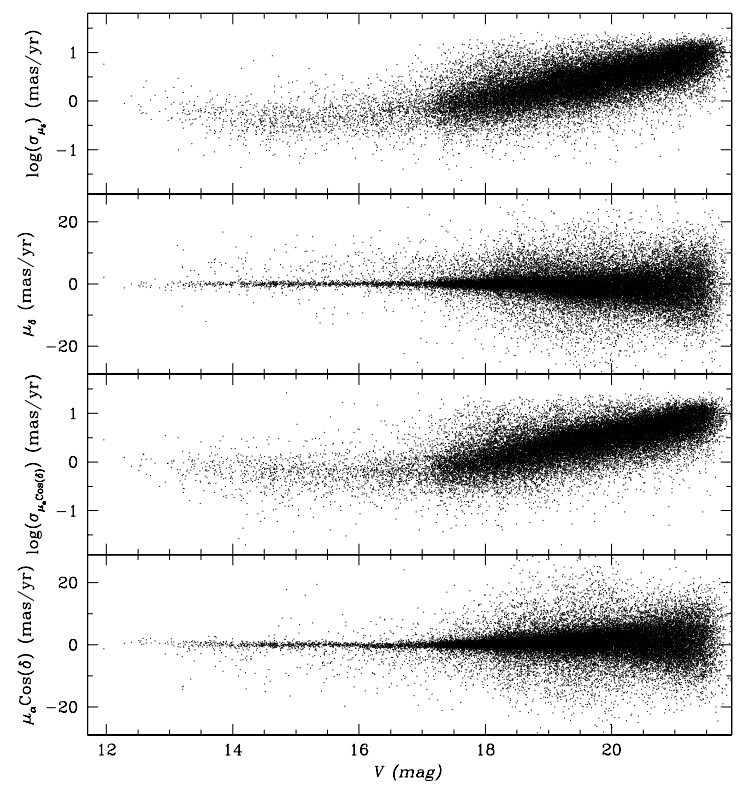

Fig. 3. Plots of proper motions and log of their standard deviations of the mean versus visual magnitude in mas $\mathrm{yr}^{-1}$.
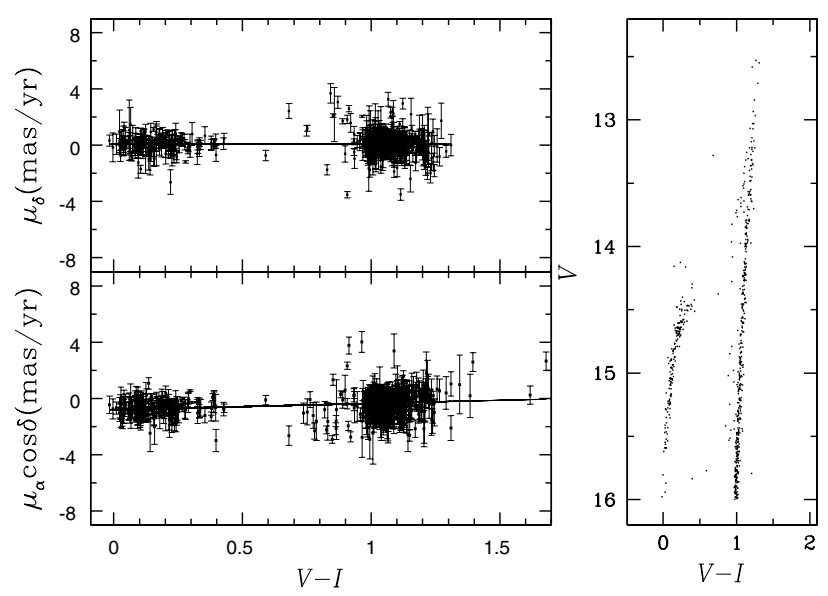

Fig. 4. Distribution of PMs as a function of colour for stars brighter than $V<16$ mag is modelled with a linear fit.

\subsubsection{Differential chromatic refraction}

Because of the wavelength dependence of the refractive index of air, a star observed with a blue filter will appear slightly higher in the sky than the same star observed through a red filter. Unfortunately, the data do not contain exposures suitable to properly correct this effect. We can, however, remove possible differences in the average differential chromatic refraction (DCR) between the two epochs.

Figure 4 shows the proper motion $\mu_{\alpha} \cos (\delta)$ and $\mu_{\delta}$ as a function of $(V-I)$ colour of horizontal and giant branch stars. These selected stars are brighter than 16th mag in $V$ and have a

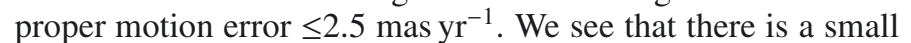
colour-related displacement of $\sim-0.81 \mathrm{mas} \mathrm{yr}^{-1}(V-I)^{-1}$ in $\mu_{\alpha} \cos (\delta)$ direction. Our final proper motion data were corrected for the DCR effect by applying the colour term correction in the proper motions.

\subsection{Cluster $C M D$ decontamination}

The vector-point diagram (VPD) of our PM measurements is shown in the top panels of Fig. 5 while $V$ vs. $(V-I)$ CMD in the bottom panels. Left panels show all stars while middle and right panels show the probable cluster members and field

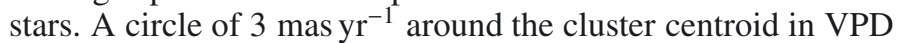
of proper motions defines our membership criterion. The chosen radius is a compromise between losing cluster members with poor proper motions and including field stars that share the cluster mean proper motion. The shape of the cluster members' PM dispersion is round, providing that our PM measurements are not affected by any systematics. The right lower panel represents the CMD for field stars. A few cluster members are also visible in this CMD because of their poorly determined proper motions. In Fig. 6, we show the $(V-I), V$ CMD which is binned along the magnitude axis. In each bin we adopted different selection criteria to identify cluster members, which were more stringent for stars with more reliable measurements from data of high signal-to-noise ratio, and less restrictive for stars with less precise measurements. The proper motion error is $<1.5 \mathrm{mas} \mathrm{yr}^{-1}$

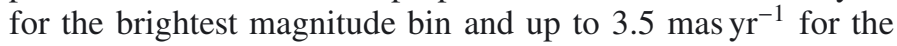
faintest bin. This figure shows that fainter stars have a stronger error in proper motion. The bright stars from the short exposures (40 s) in epoch 2 have saturated in the long exposures (200 s) of epoch 1 . The separation of brighter cluster members from the field stars is clearly visible while fainter members are not clearly separated out as seen in Fig. 6. The reason may be that proper motions for fainter stars are not determined accurately.

\subsection{Comparison with previous proper motion studies}

Absolute proper motion can be determined using galaxies observed in the field of NGC 6809. Few background faint galaxies are visible in our images, but they are not suitable to determine absolute proper motions because of the large error in their proper motions.

The absolute proper motions of stars brighter than $V=$ 18 mag are available in the UCAC3 catalogue (Zacharias et al. 2010). There are 310 common stars found to be brighter than $V=18 \mathrm{mag}$. To compare our proper motions with UCAC3, we changed the UCAC3 proper motions to relative proper motions. For this, we subtracted the absolute proper motion calcu-

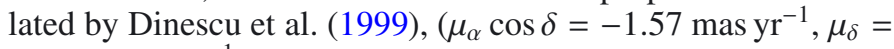
-10.14 mas $^{-1}$ ) from the individual proper motion of UCAC3. We considered the value of the absolute proper motion for the cluster by Dinescu et al. (1999) because it is more precise than the derived mean absolute proper motion using UCAC3 stars. Figure 7 shows the comparison of our PMs with those of the UCAC3 catalogue. The top-left panel shows the VPD of UCAC3 stars while the top-right panel shows the VPD of our measurements. A concentration of stars around $(0,0)$ mas $\mathrm{yr}^{-1}$ is seen in both VPDs. Our proper motions distribution is tighter than the UCAC3 distribution. This is because our data are more precise than the UCAC 3 data.

In the lower panels of Fig. 7 we show the difference $(\Delta)$ in $\mu_{\alpha} \cos \delta$ and $\mu_{\delta}$ in the sense of our minus the UCAC 3 difference as a function of $V$ magnitude. There is no systematic trend in the differences with magnitude. The $3 \sigma$ clipped median of differences are $-0.10(\sigma=3.70)$ mas yr$^{-1}$ and 2.60( $\sigma=$ 4.45) mas $\mathrm{yr}^{-1}$ in $\mu_{\alpha} \cos \delta$ and $\mu_{\delta}$.

Figure 8 shows the difference between our proper motions and Z111 plotted with $V$ magnitude. The $3 \sigma$ clipped median of differences are $-0.28(\sigma=0.86) \mathrm{mas} \mathrm{yr}^{-1}$ and $-0.02(\sigma=$ $0.82)$ mas $^{-1}$. Clearly, our measurements are consistent with the Z111 data for $V \leq 19 \mathrm{mag}$. 


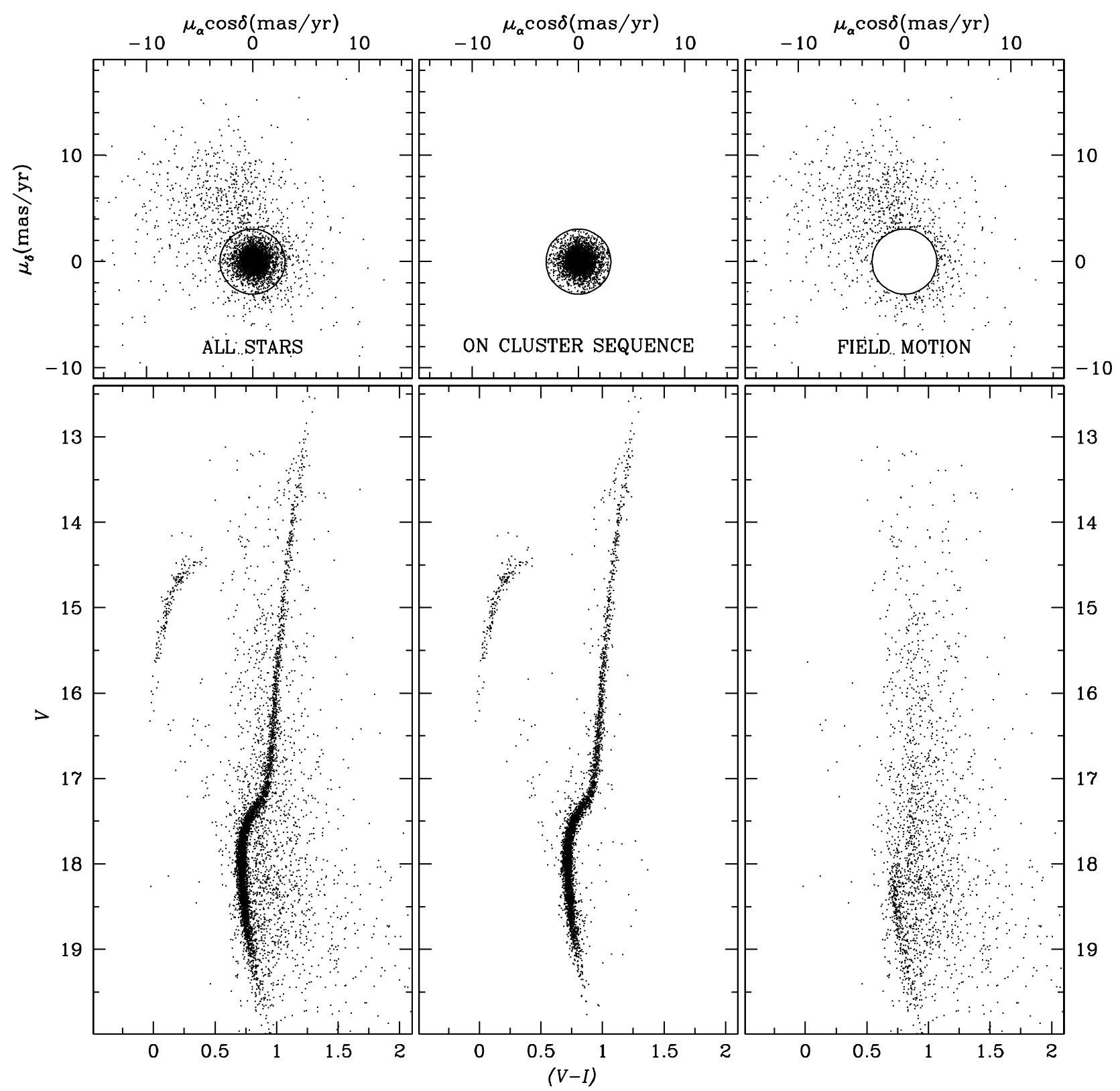

Fig. 5. (Top panels) Proper motion vector-point diagram. Zero point in VPD is the mean motion of cluster stars. (Bottom panels) Calibrated $V$

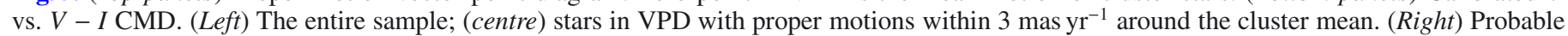
background/foreground field stars in the area of NGC 6809 studied in this paper. All plots show only stars with proper motion $\sigma$ smaller than

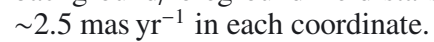

\section{Determination of membership probability}

Accurate membership determination is essential for additional astrophysical studies of cluster. The fundamental mathematical model set up by Vasilevskis et al. (1958) and the technique based upon the maximum likelihood principle developed by Sanders (1971) for membership determination have since been continuously refined.

An improved method for membership determination of stars in clusters based on proper motions with different observed precisions was developed by Stetson (1980) and Zhao \& He (1990). Zhao \& Shao (1994) then added the correlation coefficient of the field star distribution to the set of parameters describing their distribution on the sky.

The VPD in the top left panel of Fig. 5 shows the two populations. A tight clump at $\mu_{\alpha} \cos \delta=\mu_{\delta}=0.0 \mathrm{mas} \mathrm{yr}^{-1}$ represents the cluster stars and a broad distribution of field stars is centred around $(-1.71,4.14)$ mas $^{-1} r^{-1}$. To determine the membership probability, we adopted the method described in Balaguer-Nunez et al. (1998). This method has already been used for $\omega$ Centauri (Bellini et al. 2009). According to this method, first we constructed the frequency distribution of cluster stars $\left(\phi_{\mathrm{c}}^{\nu}\right)$ and field stars $\left(\phi_{\mathrm{f}}^{\nu}\right)$. The frequency function for the $i$ th star of a cluster can be written as follows:

$$
\begin{aligned}
\phi_{\mathrm{c}}^{v}= & \frac{1}{2 \pi \sqrt{\left(\sigma_{\mathrm{c}}^{2}+\epsilon_{x i}^{2}\right)\left(\sigma_{\mathrm{c}}^{2}+\epsilon_{y i}^{2}\right)}} \\
& \times \exp \left\{-\frac{1}{2}\left[\frac{\left(\mu_{x i}-\mu_{x c}\right)^{2}}{\sigma_{\mathrm{c}}^{2}+\epsilon_{x i}^{2}}+\frac{\left(\mu_{y i}-\mu_{y c}\right)^{2}}{\sigma_{\mathrm{c}}^{2}+\epsilon_{y i}^{2}}\right]\right\},
\end{aligned}
$$




\section{NGC 6809 (M 55) PM SELECTION}
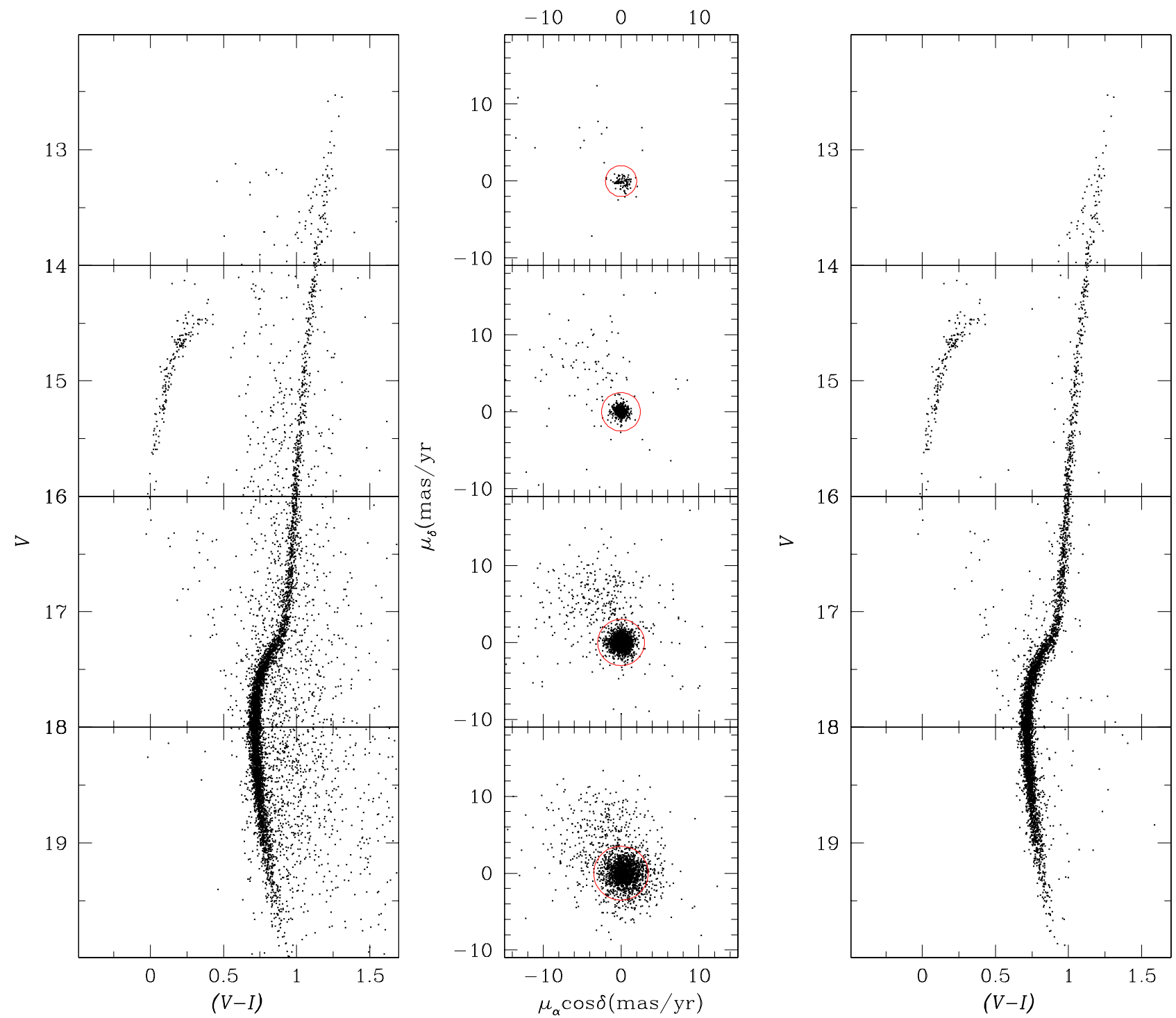

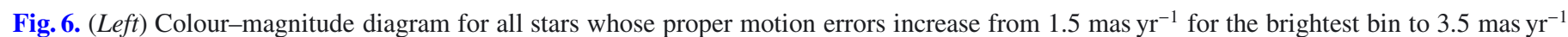
for the faintest one. (Middle) Vector-point diagram for the same stars in corresponding magnitude intervals. A circle in each plot shows the adopted membership criterion. (Right) Colour-magnitude diagram for stars assumed to be cluster members.

where $\mu_{x i}$ and $\mu_{y i}$ are the proper motions of the $i$ th star while $\mu_{x c}$ and $\mu_{y c}$ are the cluster's proper motion centre. $\sigma_{\mathrm{c}}$ is the intrinsic proper motion dispersion of cluster member stars and $\left(\epsilon_{x i}, \epsilon_{y i}\right)$ are the observed errors in the proper motion components of $i$ th star. The frequency distribution for $i$ th field star is as follows:

$$
\begin{aligned}
\phi_{\mathrm{f}}^{v}= & \frac{1}{2 \pi \sqrt{\left(1-\gamma^{2}\right)} \sqrt{\left(\sigma_{x f}^{2}+\epsilon_{x i}^{2}\right)\left(\sigma_{y f}^{2}+\epsilon_{y i}^{2}\right)}} \exp \left\{-\frac{1}{2\left(1-\gamma^{2}\right)}\right. \\
& \left.\times\left[\frac{\left(\mu_{x i}-\mu_{x f}\right)^{2}}{\sigma_{x f}^{2}+\epsilon_{x i}^{2}}-\frac{2 \gamma\left(\mu_{x i}-\mu_{x f}\right)\left(\mu_{y i}-\mu_{y f}\right)}{\sqrt{\left(\sigma_{x f}^{2}+\epsilon_{x i}^{2}\right)\left(\sigma_{y f}^{2}+\epsilon_{y i}^{2}\right)}}+\frac{\left(\mu_{y i}-\mu_{y f}\right)^{2}}{\sigma_{y f}^{2}+\epsilon_{y i}^{2}}\right]\right\},
\end{aligned}
$$

where $\mu_{x i}$ and $\mu_{y i}$ are the proper motions of $i$ th star while $\mu_{x f}$ and $\mu_{y f}$ are the field proper motion centre. $\epsilon_{x i}$ and $\epsilon_{y i}$ are the observed errors in proper motions component and $\sigma_{x f}$ and $\sigma_{y f}$ are the field intrinsic proper motion dispersions and $\gamma$ is the correlation coefficient. $\gamma$ can be calculated as

$\gamma=\frac{\left(\mu_{x i}-\mu_{x f}\right)\left(\mu_{y i}-\mu_{y f}\right)}{\sigma_{x f} \sigma_{y f}}$,

owing to the small observed field of the cluster we did not consider the spatial distribution of the stars. To define the distribution function $\phi_{\mathrm{c}}^{v}$ and $\phi_{\mathrm{f}}^{v}$, we used stars with a proper motion error

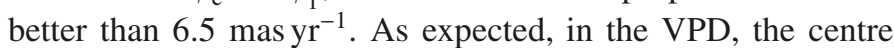
of cluster stars is found to be at $\mu_{x c}=0.0 \mathrm{mas} \mathrm{yr}^{-1}$ and $\mu_{y c}=$ $0.0 \mathrm{mas} \mathrm{yr}^{-1}$. Our proper motion data set could not determine intrinsic proper motion dispersion $\left(\sigma_{\mathrm{c}}\right)$ for cluster stars. Therefore, we adopted $\sigma_{\mathrm{c}}=0.2 \mathrm{mas} \mathrm{yr}^{-1}$ as calculated in Sect. 2.1.3. For field stars, we have $\mu_{x f}=-1.71 \mathrm{mas} \mathrm{yr}^{-1}, \mu_{y f}=4.14 \mathrm{mas} \mathrm{yr}^{-1}$, $\sigma_{x f}=4.56$ mas yr$^{-1}$ and $\sigma_{y f}=4.37 \mathrm{mas} \mathrm{yr}^{-1}$.

The distribution of all the stars can be calculated as $\phi=\left(n_{\mathrm{c}} \cdot \phi_{\mathrm{c}}^{v}\right)+\left(n_{\mathrm{f}} \cdot \phi_{\mathrm{f}}^{v}\right)$, 

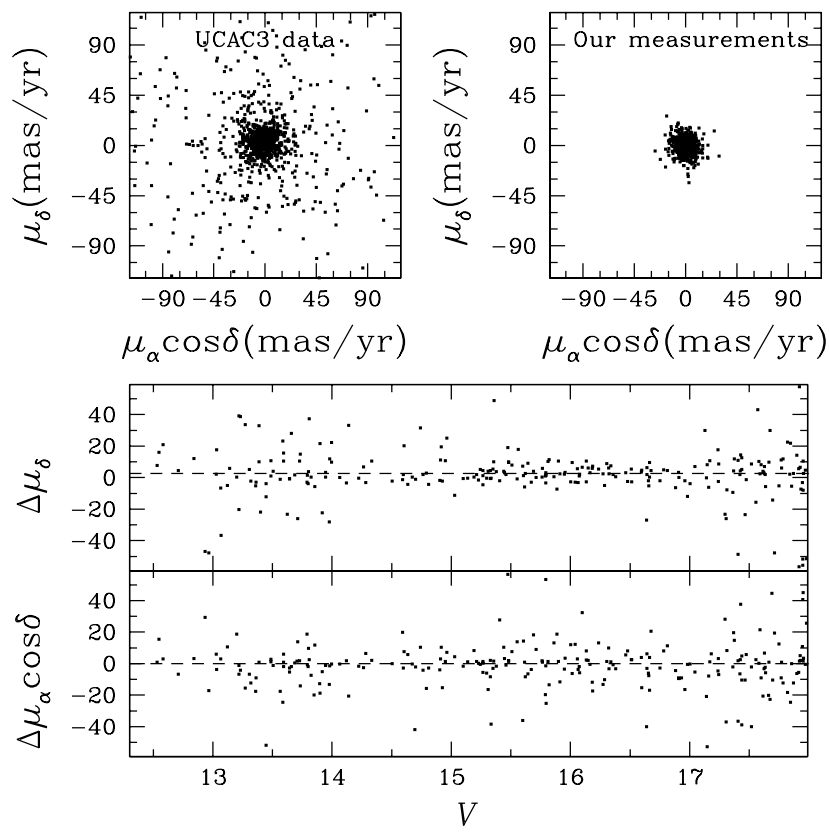

Fig. 7. Top panels: vector-point diagrams of common stars relative to the cluster mean motion for UCAC3 (left) and our catalogue (right). (Bottom panels) right ascension (bottom) and declination (top) proper motion differences as a function of $V$ magnitude between UCAC3 and our measurements. Horizontal dashed lines show the $3 \sigma$-clipped median of the proper motion difference between UCAC 3 and our data.

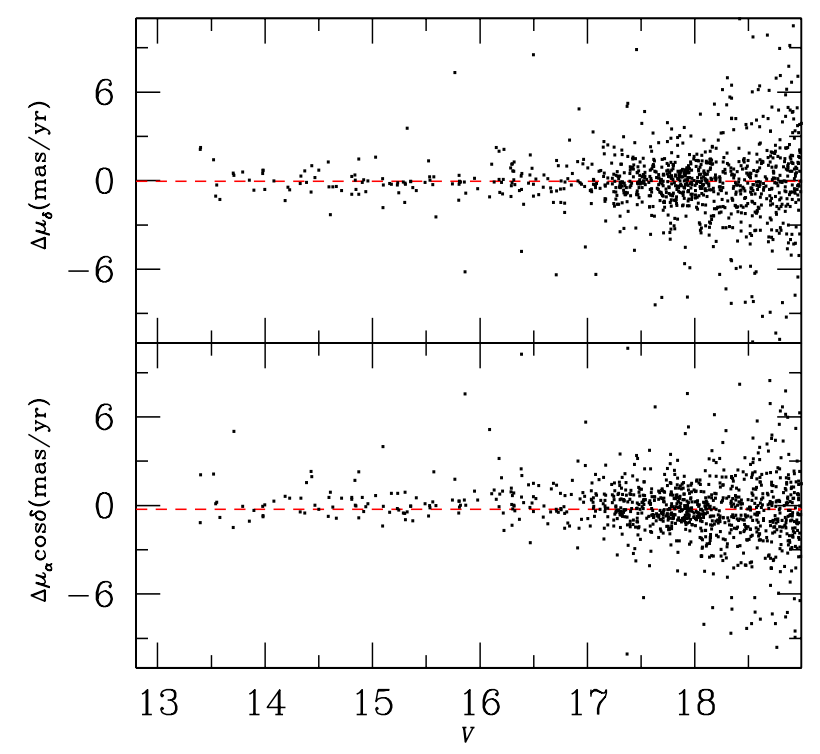

Fig. 8. Plot of proper motion differences between Z111 and our data with $V$ magnitude. Horizontal dashed lines show the $3 \sigma$-clipped median of the proper motion difference between Z111 and our data.

where $n_{\mathrm{c}}$ and $n_{\mathrm{f}}$ are the normalized number of stars for cluster and field $\left(n_{\mathrm{c}}+n_{\mathrm{f}}=1\right)$. Therefore, the membership probability for $i$ th star is

$P_{\mu}(i)=\frac{\phi_{\mathrm{c}}(i)}{\phi(i)}$

a good indicator of cluster and field separation is the membership probability. It is plotted as a function of magnitude in Fig. 9. As seen in this plot, high membership probability $\left(P_{\mu}>90 \%\right)$ extend down to $V \sim 19$ mag. At fainter magnitudes the membership probability gradually decreases.

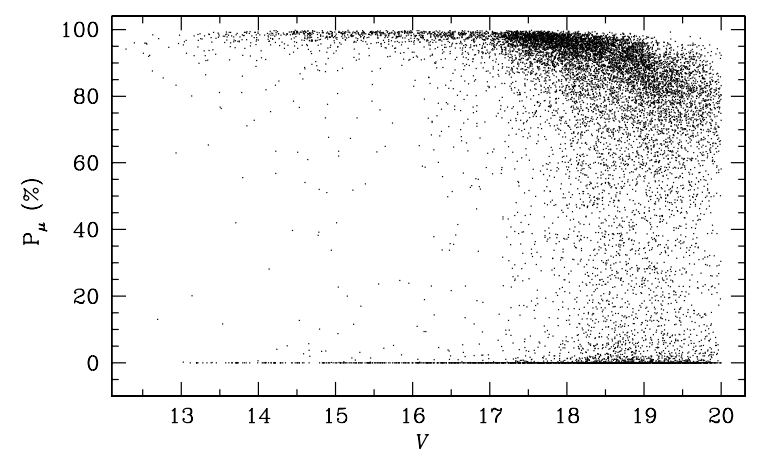

Fig. 9. Membership probability $P_{\mu}(\%)$ as a function of the $V$ magnitude for all stars in our catalogue. At $V \sim 18.5 \mathrm{mag}$ and fainter, $P_{\mu}$ diminishes as a result of increasing errors in the PMs.

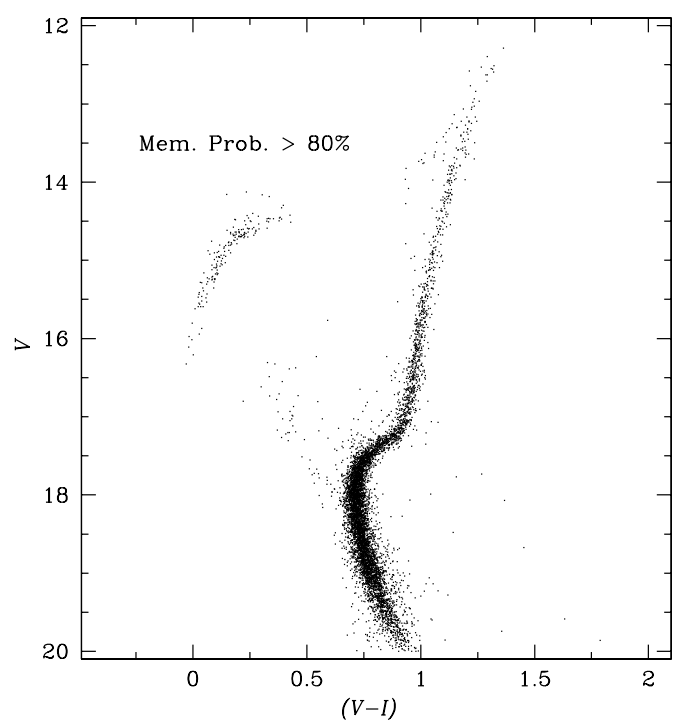

Fig. 10. Colour-magnitude diagram for the stars with membership probability $>80 \%$.

Figure 10 shows the CMD with membership probability $P_{\mu}>80 \%$. This CMD shows a clean main-sequence down to $V \sim 20$ mag and distinct population of sub-giants, red giants, horizontal branch stars, and blue stragglers.

\section{Applications}

\subsection{Membership probability of variables in NGC 6809}

Our catalogue can be used to assign membership probability of many variables stars in this cluster. Olech et al. (1999) have found 15 RR Lyrae photometric variables using the data taken with $1.0 \mathrm{~m}$ Swope telescope at Las Campanas Observatory. One star is found in our catalogue. Its membership probability is $P_{\mu}=77 \%$ and it is listed in Table 3. Our $P_{\mu}$ value shows that this star is a cluster member. NGC 6809 is known to have a large population of SX Phoenicis (SX Phe) variables, which are found in the BSS region shown in Pych et al. (2001). Out of 27, four are found in our catalogue and their membership probabilities are also listed in Table 3. All four of the SX Phe stars have a membership probability higher than $96 \%$ and are confirmed cluster members. Bassa et al. (2008) found 31 X-ray sources in the direction of NGC 6809. Out of 31 sources, 23 are found in our catalogue and are listed in Table 4 . Out of these 23 sources, 18 have a membership probability higher than $68.27 \%$. These 18 X-ray sources may be cluster members. X-ray sources CX2, CX17, CX23, and CX26 have membership probability less than $20 \%$. 
Table 3. Membership probability for RR lyrae variables provided by Olech et al. (1999) (ID $)$ and SX Phoenicis variables given by Pych et al. (2001).

\begin{tabular}{cccccc}
\hline \hline & RR Lyrae & & \multicolumn{3}{c}{ SX Phoenicis } \\
ID $_{0}$ & $P_{\mu}$ & ID & $\mathrm{ID}_{p}$ & $\begin{array}{c}P_{\mu} \\
(\%)\end{array}$ & ID \\
& $(\%)$ & & & 96 & 9246 \\
\hline V5 & 77 & 5098 & V24 & 97 & 10157 \\
& & & V35 & 97 & 5754 \\
& & & V38 & 97 & 9541 \\
\hline
\end{tabular}

Notes. ID refers our catalogue number.

Table 4. Membership probability for blue stragglers given by Lanzoni et al. (2007) (ID $)$ and X-ray sources given by Bassa et al. (2008) (ID $\mathrm{I}_{\mathrm{b}}$.

\begin{tabular}{lccccc}
\hline \hline Bing & Blue stragglers & & \multicolumn{3}{c}{ X-ray sources } \\
ID & $P_{\mu}$ & ID & ID $_{\mathrm{b}}$ & $\begin{array}{c}P_{\mu} \\
(\%)\end{array}$ & ID \\
& $(\%)$ & & & 13 & 8855 \\
\hline BSS4 & 93 & 7781 & CX2 & 91 & 7889 \\
BSS11 & 95 & 6120 & CX3 & 96 & 7507 \\
BSS16 & 39 & 6783 & CX4 & 86 & 8478 \\
BSS35 & 30 & 8518 & CX5 & 98 & 3186 \\
BSS39 & 98 & 11625 & CX6 & 98 \\
BSS42 & 32 & 2010 & CX7 & 94 & 4760 \\
BSS51 & 95 & 3419 & CX8 & 93 & 4460 \\
BSS56 & 74 & 7921 & CX9 & 95 & 7287 \\
BSS57 & 97 & 9246 & CX11 & 74 & 6605 \\
& & & CX12 & 98 & 8594 \\
& & & CX13 & 57 & 4158 \\
& & & CX15 & 95 & 9803 \\
& & & CX16 & 94 & 7222 \\
& & CX17 & 00 & 7125 \\
& & CX19 & 83 & 5189 \\
& & CX20 & 98 & 6856 \\
& & CX21 & 83 & 2046 \\
& & CX23 & 05 & 9983 \\
& & CX24 & 76 & 1563 \\
& & CX26 & 00 & 2266 \\
& & CX27 & 96 & 1645 \\
& & CX28 & 96 & 1117 \\
& & CX30 & 86 & 6286 \\
\hline
\end{tabular}

Notes. ID refers our catalogue number.

Hence, they may not be cluster members. Membership probability of CX17, CX23, and CX26 is $0 \%$, which means that they are field-region objects.

Lanzoni et al. (2007) have studied 65 blue straggler populations by using high-resolution HST and wide-field groundbased observations in ultraviolet and optical bands. Nine objects match with our catalogue and are listed in Table 4. Out of these nine, six have $P_{\mu}>73 \%$ and three have $P_{\mu}<40 \%$. Hence, six blue stragglers are associated with the cluster and three may be field objects. All RR Lyrae variables, SX Phe stars, X-ray sources, and blue stragglers are shown in the colour-magnitude diagram with different symbols in Fig. 11.

\section{The catalogue}

The electronic catalogue is available at the CDS and also by request to the authors. First few lines of the electronic catalogue are given in Table 5. In the catalogue, Col. (1) contains the running number; Cols. (2) and (3) provide the J2000 equatorial coordinates in degree, while Cols. (4) and (5) provide

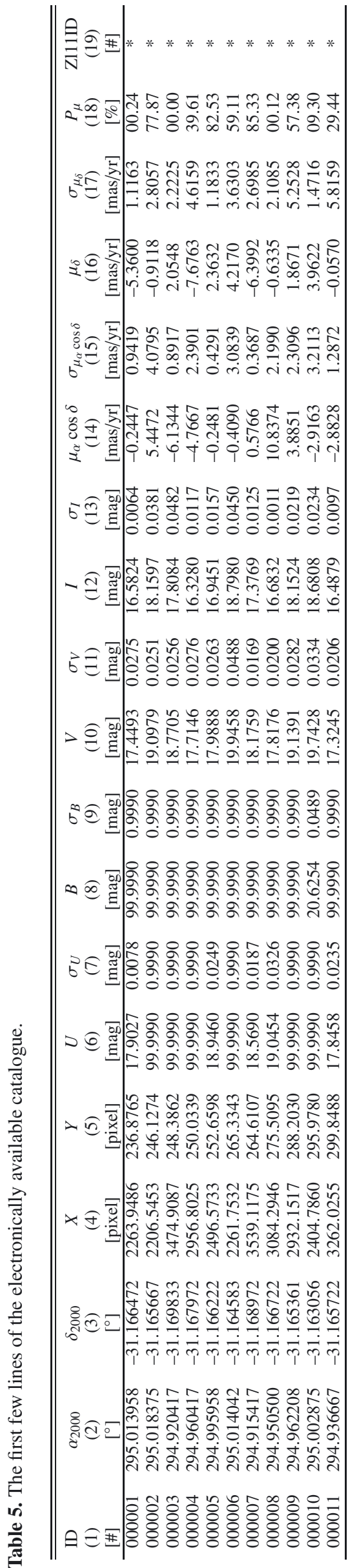




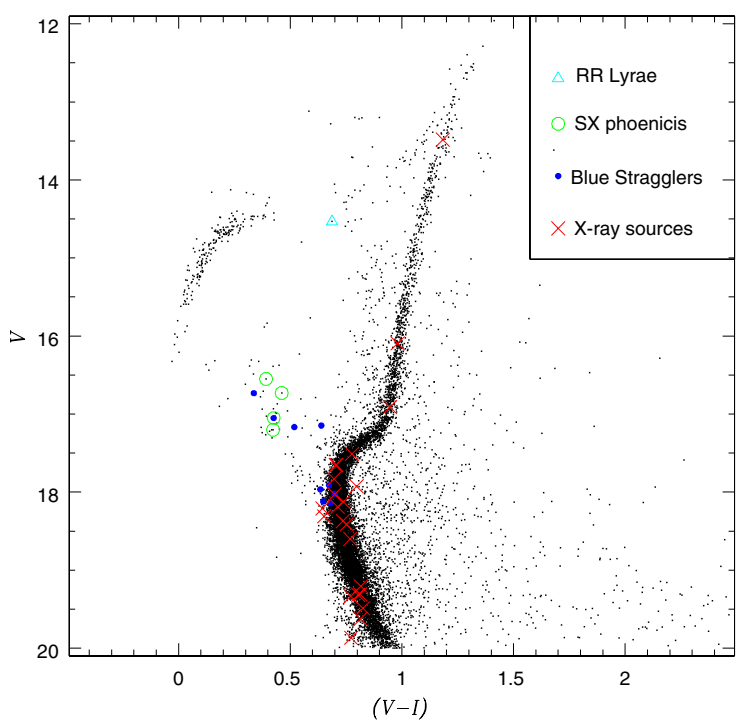

Fig. 11. Colour-magnitude diagram for the stars whose membership probability has been calculated. All variables, blue stragglers, and X-ray sources listed in Tables 3 and 4 are shown with different symbols indicated in the inset.

the pixel coordinates $X$ and $Y$ in a distortion-corrected reference frame. Columns (6) to (13) give photometric data, i.e., $U, B, V$ and $I$ magnitudes and their corresponding errors. If photometry in a specific band is not available, a flag equal to 99.999 is set for the magnitude and 0.999 for the error. Columns (14) to (17) represent relative proper motions and their standard errors in mas $\mathrm{yr}^{-1}$. Column (18) gives the membership probability $P_{\mu}(\%)$. The last Col. (19) gives the ID number of Z111.

\section{Conclusions}

We provided a catalogue of precise proper motions and membership probability of stars in the wide-field region of globular cluster NGC 6809. We have obtained precise proper motions and astrometric membership probabilities down to $V \sim 20 \mathrm{mag}$ in $26 \times 22 \operatorname{arcmin}^{2}$ area around the globular cluster NGC 6809 . Finally, we provided the membership probability for different types of variable stars, blue stragglers, and X-ray sources. We also demonstrated that the CCD observations taken just seven years apart can provide accurate proper motions. These proper motions are used to separate the cluster members from field stars down to $V \sim 20 \mathrm{mag}$.

Acknowledgements. We are grateful to the referee Floor van Leeuwen for his careful reading of the manuscript and many useful suggestions. We also thank Dr. J. Kaluzny for providing us $U$ filter data for our calibration purpose. This research used the facilities of the Canadian Astronomy Data Centre operated by the National Research Council of Canada with the support of the Canadian Space Agency.

\section{References}

Anderson, J., Bedin, L. R., Piotto, G., Yadav, R. K. S., \& Bellini, A. 2006, A\&A, 454, 1029 (Paper I)

Bassa, C. G., Pooley, D., Verbunt, F., et al. 2008, A\&A, 488, 921

Bellini, A., Piotto, G., Bedin, L. R., et al. 2009, A\&A, 493, 959

Balaguer-Núñez, L., Tian, K. P., \& Zhao, J. L. 1998, A\&AS, 133, 387

Dinescu, D. I., van Altena, W. F., \& Girard, T. M. 1999, AJ, 117, 277

Harris, W. E. 1996, AJ, 112, 1487

Kaluzny, J., Pietrukowicz, P., Thompson, I. B., et al. 2005, MNRAS, 359, 677

Lanzoni, B., Dalessandro, E., Perina, S., et al. 2007, ApJ, 670, 1065

Olech, A., Kaluzny, J., Thompson, I. B., et al. 1999, AJ, 118, 442

Pryor, C., \& Meylan, G. 1993, ASP Conf. Ser., 50, 357

Pych, W., Kaluzny, J., Krzeminski1, W., Schwarzenberg-Czerny, A., \& Thompson, I. B. 2001, A\&A, 367, 148

Sanders, W. L. 1971, A\&A, 14, 226

Stetson, P. B. 1980, AJ, 85, 387

Vargas Álvarez, C. A., \& Sandquist, E. L. 2007, AJ, 134, 825

Vasilevskis, S., Klemola, A., \& Preston, G. 1958, AJ, 63, 387

Yadav, R. K. S., Bedin, L. R., Piotto, G., et al. 2008, A\&A, 484, 609

Zacharias, N., Finch, C., Girard, T., et al. 2010, AJ, 139, 2184

Zhao, J. L., \& He, Y. P. 1990, A\&A, 237, 54

Zhao, J. L., \& Shao, Z. Y. 1994, A\&A, 288, 89

Zloczewski, K., Kaluzny, J., \& Thompson, I. B. 2011, MNRAS, 414, 3711 